Bull. Mater. Sci., Vol. 6, No. 3, July 1984, pp. 611-616. (C) Printed in India.

\title{
Electron diffraction analysis of antimony films
}

\author{
P D PRABHAWALKER and AMARJIT SINGH* \\ Regional Sophisticated Instrumentation Centre Indian Institute of Technology, Powai, \\ Bombay 400076 , India \\ * Department of Physics, University of Bombay, Vidyanagari, Bombay 400098, India
}

\begin{abstract}
Antimony films of about $3000 \AA$ thickness are grown on $\mathrm{NaCl}$ substrate at various substrate temperatures. The films were subsequently analysed for electron diffraction and for grain size on electron microscope. At lower substrate temperatures, the film showed a single crystalline growth, and the crystal grain size was small. However at higher substrate temperatures a cubic phase along with hep phase was found to exist and also the enlargement of the grain size was observed.
\end{abstract}

Keywords. Electron diffraction; antimony film; metastable crystalline states; phase changes.

\section{Introduction}

Recently the studies of semi-metal films particularly antimony films, have been a matter of considerable interest. Antimony films exhibit interesting thermoelectric properties (Boyer et al 1981), and also crystallographically, a number of metastable crystalline states are observed in liquid quenched or vapour-deposited antimony films (Dilshad Akhter et al 1979). Antimony is known to exist in a simple cubic structure $(a=2 \cdot 96)$, and in hcp structure $(a=3.33 \AA$ and $c / a=1.58)$ at pressures of 50 and $90 \mathrm{kbar}$ respectively (Kabalkina and Mylov 1964). In the present work, a systematic study on antimony films deposited at various substrate temperatures has been carried out. Its subsequent effect on the microstructure and the crystal structure of antimony films has been studied by electron microscopy.

\section{Experimental}

Antimony of high purity $(99.99 \%)$ was evaporated from a tungsten boat at a pressure $2 \times 10^{-6}$ torr. Initially an attempt was made to use glass as a substrate, however it was rather inconvenient to remove thin antimony films of the order of $300 \AA$ from the substrate. Hence compressed sodium chloride pellets were prepared and they were transferred immediately from the desiccator to the evaporater. The pellets were kept in close contact with the substrate heater. The thickness of the films was estimated from the amount of antimony evaporated as well as by a thickness monitor. It was ensured that the deposition took place on dry pellet at the desired temperature. After the deposition was complete, the thin films were taken out by dissolving the pellet in water. The thin films were examined on a Phillips-EM 301 transmission electron microscope. The microscope was operated at $60 \mathrm{kV}$ accelerating potential and at magnification of $\times 40,000$. 


\section{Results}

For the sake of better comparison, the same accelerating voltage has been maintained $(60 \mathrm{kV})$ throughout the experiment, which gives the wavelength of the incident radiation as $0.0487 \AA$. Since the same camera constant is used, it is possible to compare the spots/rings of one figure with that of the other.

Figures la,b show the electron micrograph and the electron diffraction pattern respectively of the film deposited at room temperature. The electron micrograph shows formation of small granular structures indicating formation of crystalline state. The electron diffraction pattern had a number of rings. The diameter of these rings given in table 1 shows that the ratios of $d^{2}$ for the outer ring to the next ring in order is a constant

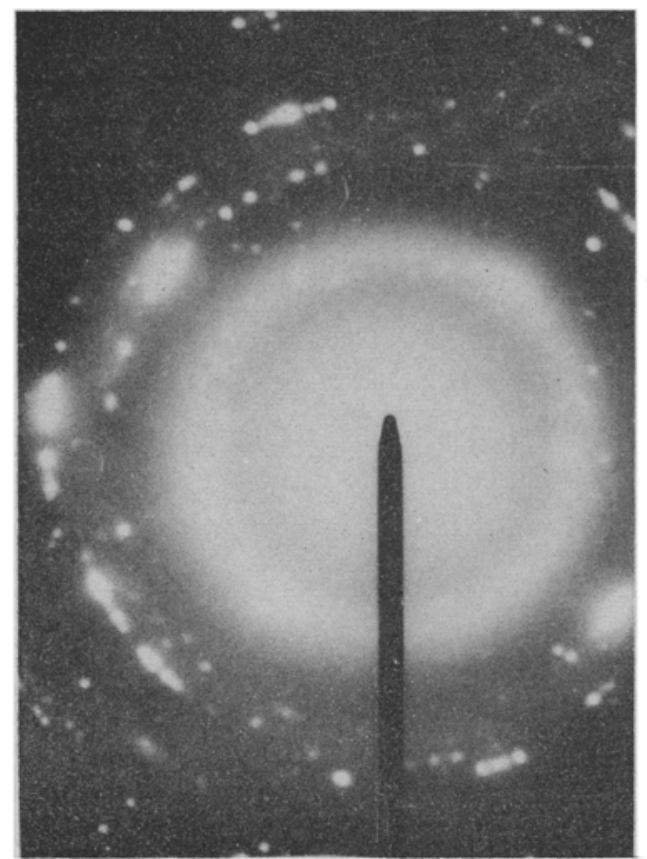

(a)

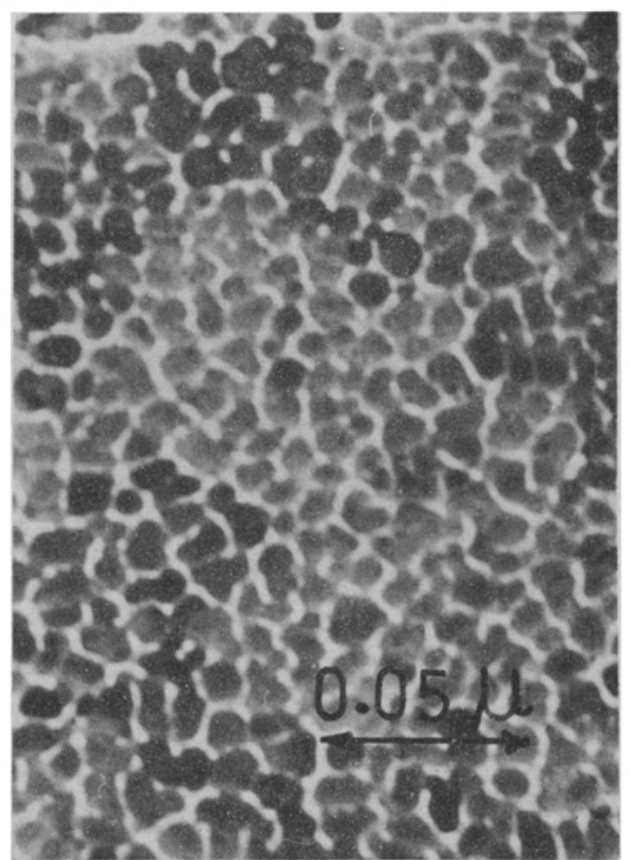

(b)

Figure 1. a. Electron diffraction pattern b. Electron micrograph of the antimony film deposited at room temperature

Table 1. Diameter of electron diffraction rings and the ratio of their squares, substrate being at room temperature.

\begin{tabular}{cccc}
\hline Ring No. $\quad d_{n}=\begin{array}{c}\text { diameter of } \\
\text { the ring } n\end{array}$ & $d_{n}^{2} \quad \sum=\left(d_{n} / d_{n-1}\right)^{2}$ \\
\hline 1 & 3.07 & 9.43 & - \\
2 & 3.92 & 15.37 & 1.63 \\
3 & 4.94 & 24.40 & 1.59 \\
4 & 6.22 & 38.69 & 1.59 \\
5 & 7.83 & 61.31 & 1.59 \\
\hline
\end{tabular}


1.59. Reference to the camera geometry and Bunn charts reveals that the structure is hep and the distance corresponds to $(10 \overline{10})$ plane. The constants of the hcp crystal were according to the previous literature, i.e. $a=3.33 \AA$ and $c / a=1.58$ (Dilshad Akhter et al 1979).

Figures $2 \mathrm{a}, \mathrm{b}$ show the diffraction patterns and the electron micrograph respectively of the films deposited at $323 \mathrm{~K}$. It has been observed that the grain size has become larger and the crystal structure has started changing. The electron diffraction pattern corresponds to a spot pattern showing (110) planes of a cubic structure and some traces of the previous ring pattern are also seen.

At further elevated temperatures i.e. at $373 \mathrm{~K}$, the cubic pattern becomes predominant and large crystals are formed. The previous ring pattern is faintly visible (figures $3 \mathrm{a}, \mathrm{b}$ ).

At $423 \mathrm{~K}$ the pattern becomes more complicated and absolutely polycrystalline (figures $4 \mathrm{a}, \mathrm{b}$ ). We get a number of rings in the diffraction pattern. Nine rings which were predominantly seen were taken for comparison. Starting from the outermost ring, squares of the ratios of the diameters of the inner ones were taken and are shown in table 2.

A close look at the table shows that the ratios $9 / 7,8 / 6$, and $6 / 4$ are around $1 \cdot 59$. Since the magnification, incident radiation and camera constant have remained the same, it can be concluded that these rings resemble the previous hcp structure with $a=3.33 \AA$ and $c / a=1 \cdot 58$. The second commonly occurring ratio is between $6 / 5,5 / 4,4 / 3$ and $9 / 8$ and is 1.12 .

Excepting these two ratios there are no more ratios which are repeating. We can

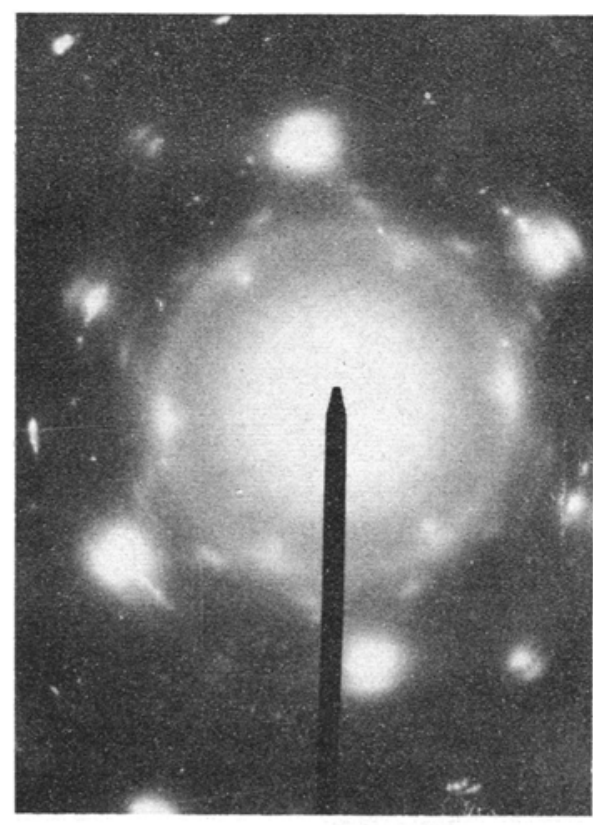

(a)

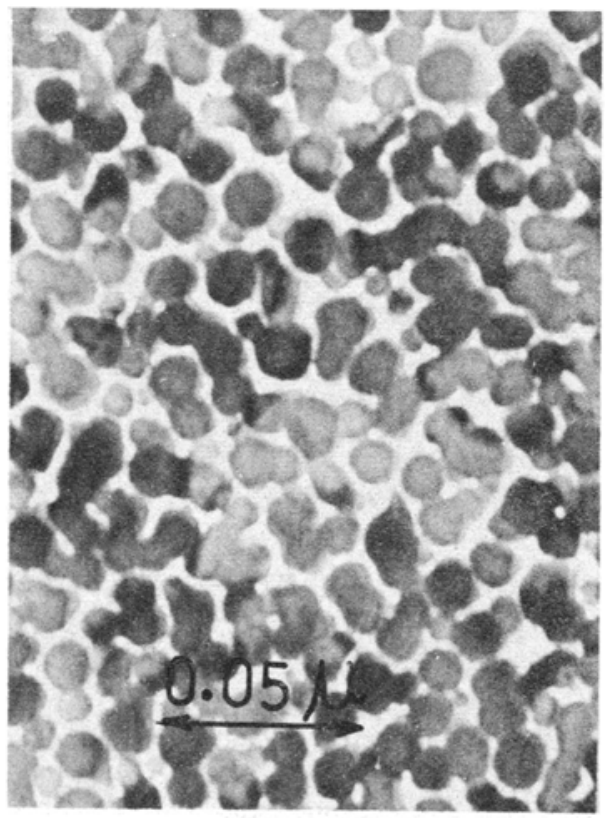

(b)

Figure 2. a. Electron diffraction pattern $\mathbf{b} .(\times 44000)$ Electron micrograph deposited at $323 \mathrm{~K}$. 


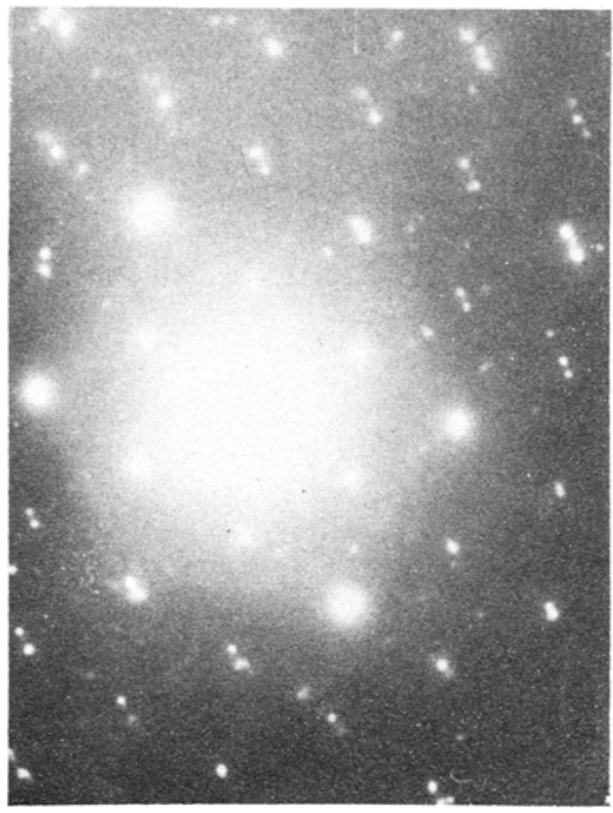

(a)

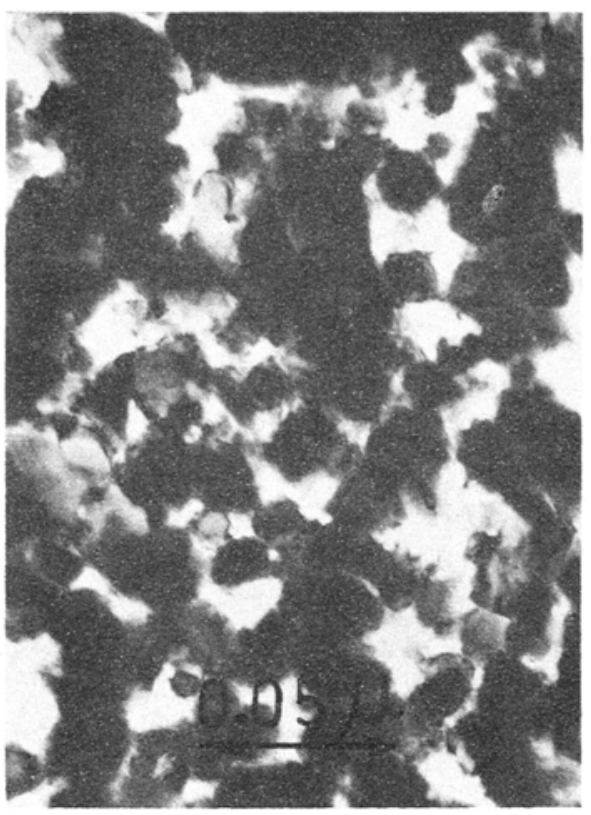

(b)

Figure 3. a. Electron diffraction pattern b. $(\times 44000)$ Electron micrograph of the film deposited at $373 \mathrm{~K}$.

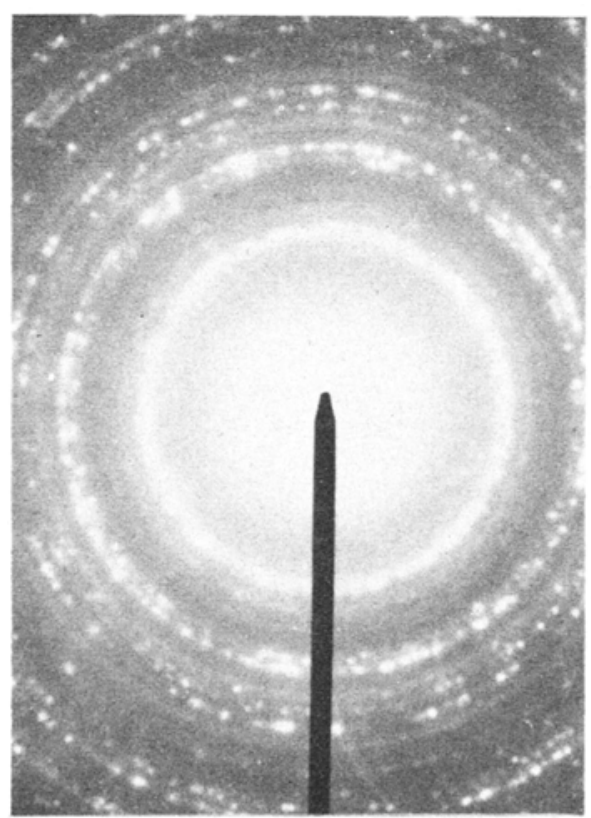

(a)

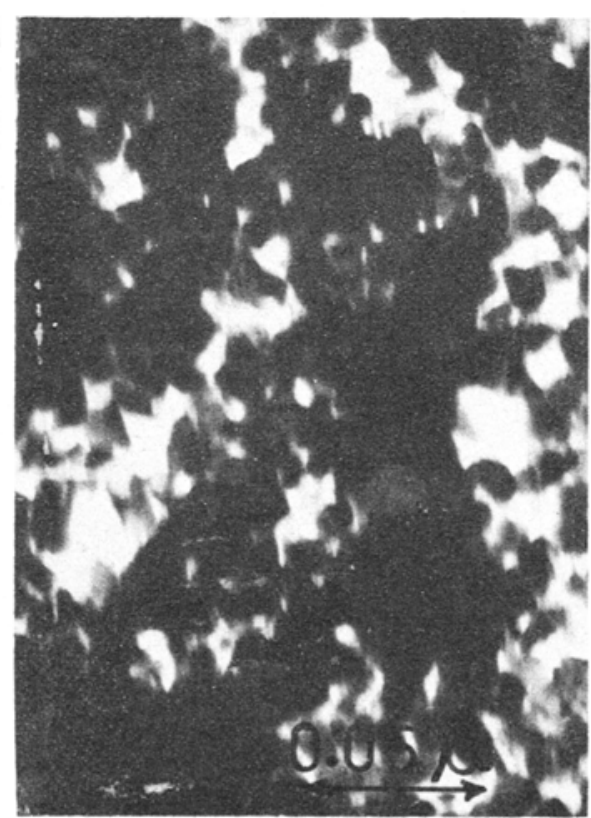

(b)

Figure 4. a. Electron diffraction pattern b. $(\times 44000)$ Electron micrograph of the film deposited at $423 \mathrm{~K}$. 
Table 2. Squares of the ratios of the outer diameter to the inner one.

\begin{tabular}{|c|c|c|c|c|c|c|c|c|}
\hline \multicolumn{3}{|c|}{ Ring No. } & \multicolumn{6}{|c|}{ Value for various ratios $\sum=\left(d_{n} / d_{N j}\right)^{2}$} \\
\hline & $N=98$ & 7 & 6 & 5 & 4 & 3 & 2 & 1 \\
\hline 9 & $-1 \cdot 14$ & 1.57 & 2.25 & $2 \cdot 48$ & $3 \cdot 11$ & 3.93 & 6.90 & 13.08 \\
\hline 8 & $\ldots$ & $1 \cdot 43$ & 1.67 & 1.84 & $2 \cdot 33$ & 2.92 & $5 \cdot 14$ & 9.73 \\
\hline 7 & $-\cdots$ & - & 1.57 & 1.98 & $2 \cdot 50$ & $3 \cdot 10$ & $4 \cdot 39$ & $8 \cdot 31$ \\
\hline 6 & -- & - & - & $1 \cdot 12$ & 1.59 & 1.75 & 2.78 & 5.27 \\
\hline 5 & -- & - & - & - & $1 \cdot 12$ & 1.45 & $2 \cdot 80$ & $5 \cdot 30$ \\
\hline 4 & -- & - & - & - & - & $1 \cdot 12$ & 1.76 & $3 \cdot 32$ \\
\hline 3 & $\ldots$ & - & - & $\ldots$ & - & - & 1.76 & $3 \cdot 32$ \\
\hline 2 & -- & -- & - & - & - & - & - & 1.89 \\
\hline
\end{tabular}

attribute this second ratio to the cubic phase seen in the previous figures. Other ratios may be due to the metastable phases of other crystals in the process of formation or might be some other reflections of the existing crystals. They were not considered as they were not significant in number.

Since the photographs were taken under the same magnification and since we know that the cubic plane is $(110)$, and the hexagonal one is (1010), the interatomic distance of the cubic phase is estimated to be $3 \cdot 4 \AA$.

\section{Discussion}

The present study shows that because of the variation of the substrate temperature a number of additional phases are developed in the vapour-deposited antimony film. If the series of the diffraction patterns are observed carefully, it is found that the sample gradually changes from a polycrystalline hep to crystalline cubic and then back to polycrystalline hcp and cubic mixture. In figure 3 the hcp structure has almost vanished. Similarly it is also expected that at some temperature between 373 and $423 \mathrm{~K}$ this hcp structure may reappear again. The study of this point is worth undertaking; however it would need a better control of substrate temperature. The cubic phase observed has a larger interatomic distance i.e. 3.4 $\AA$. In previous studies by splat cooling (Dilshad Akhter et al 1979), such a structure with $a=3.16$ was observed.

\section{Conclusions}

(i) hcp phase of antimony is observed by vapour deposition also. (ii) By increasing the substrate temperature, the cubic phase is also observed. (iii) The cubic phase has larger interatomic distances than usual. (iv) At intermediate substrate temperature hcp phase vanished and reappeared at higher temperatures. (v) At the substrate temperature of $423 \mathrm{~K}$ both hexagonal and cubic phases become polycrystalline. 


\section{Acknowledgments}

Thanks are due to Dr C S Harendranath for making the TEM facility available. The authors also thank Mr Nageshwar Rao and Miss Mruga H Joshi for help in the use of TEM.

\section{References}

Boyer A, Des B, Chacht and Groubert 1981 Thin Solid Films 76119

Dilshad Akhtar Venkar V D and Chopra K L 1979 Thin Solid films 58327

Kabalkina S S and Mylov V P 1964 Sov. Phys-Dokl 8917 